\title{
Determination and dietary risk assessment of 284 pesticide residues in local fruit cultivars in Shanghai, China
}

\section{Yaodan Zhang}

Shanghai Academy of Agricultural Sciences

\section{Wenshuai Si}

Shanghai Academy of Agricultural Sciences

\section{Lei Chen}

Shanghai Academy of Agricultural Sciences

\section{Guoqing Shen}

Shanghai Jiao Tong University

\section{Bing Bai ( $\nabla$ whitebing2@hotmail.com )}

Shanghai Academy of Agricultural Sciences

Changyan Zhou ( $\square$ zhouchangyansaas@163.com )

Shanghai Academy of Agricultural Sciences

\section{Research Article}

Keywords: Multi-residue analysis, Pesticide residues, Dietary risk assessment, UPLC-QTOF/MS, Fruit

Posted Date: March 10th, 2021

DOI: https://doi.org/10.21203/rs.3.rs-268771/v1

License: (c) (i) This work is licensed under a Creative Commons Attribution 4.0 International License.

Read Full License 


\section{Abstract}

The presence of pesticide residues has become one of the main risk factors affecting the safety and quality of agro-food. In this study, a multi-residue method for the analysis of 284 pesticides in five local fruit cultivars in Shanghai was developed based on ultrahigh-performance liquid chromatographyquadrupole time-of-fight mass spectrometry (UPLC-QTOF/MS). The limits of determination and the limits of quantitation of pesticides were $0.6-10$ and $2-30 \mu \mathrm{g} / \mathrm{kg}$, respectively. A total of $44,10,10,18$, and 7 pesticides were detected in strawberries, watermelons, melons, peaches, and grapes, respectively. The pesticide levels in $95.0 \%$ of the samples were below the maximum residual limits (MRLs) prescribed by China, and in $66.2 \%$ of the samples below the EU MRLs. The dietary risk assessment study showed big differences in the chronic and acute exposure risk values among different Chinese consumer groups. Through fruit consumption, children/females showed higher exposure risks than adults/males. But both the risk values were less than $100 \%$, indicating that potential dietary risk induced by the pesticides was not significant for Chinese consumers. Nevertheless, certain measures are needed for both growers and government in order to decrease the MRL-exceeding rate of pesticide residues and ensure the quality and safety of fruits for consumers.

\section{Introduction}

Fruits are important sources of vitamins, minerals, and beneficial phytochemicals, which have important functional properties such as anti-inflammatory, antioxidant, anti-neurodegenerative, and anticarcinogenic activities $^{1-3}$. Besides the nutritive value, fruits can also be a source of pesticide residues. In order to increase fruit yield, a variety of pesticides are used to control pests (e.g., aphids, whiteflies, mites, and nematodes) and diseases (e.g., leaf spot, powdery mildew, gray mold, verticillium wilt, and anthracnose) during the growing season ${ }^{4-7}$. As a result, pesticide residues often occur on the fresh products. According to the Environmental Working Group (EWG)'s 2020 Shopper's Guide to Pesticides in Produce, strawberries have led the ranking of the fresh fruits most contaminated with pesticide residues, followed by nectarines, apples, grapes, and peaches ${ }^{8}$. Residues of some non-recommended and restricted use pesticides can also be found in fruits due to the illegal use or from indirect sources such as soil, plastic films, neighbouring fields and crops, and irrigation waters ${ }^{9,10}$.

Exposure to pesticides may cause acute or chronic toxicity with harmful effects on human health, especially on children and pregnant women who are more vulnerable to the toxic effects ${ }^{11}$. A growing number of epidemiological and molecular studies provide evidence that pesticides have been associated with adverse health effects such as cancers, birth defects, reproductive abnormalities, toxicities, and even death $^{12-15}$. As fruits are mainly consumed fresh, and given the potential hazard of pesticide residues, the European Union (EU) and many other countries have established the maximum residue limits (MRLs) for pesticides in agricultural products to minimize pesticide residue levels and help ensure that pesticides are not overused and residues found in food are tolerable for humans ${ }^{16-21}$. The general MRLs recommended by the EU and China for pesticide residues in fruits are in the range of $0.01-10 \mathrm{mg} / \mathrm{kg}$, depending on the 
pesticides ${ }^{18,22}$. As the list of banned/restricted and authorized/registered pesticides for use on fruits is continually changing, a sensitive, accurate, and efficient analytical method is necessarily needed to detect a wide diversity of pesticide residues in fruits.

Multi-residue methods incorporate one sample preparation procedure with analytical equipment that is able to determine diverse compounds ${ }^{17,23}$. Chromatographic techniques, such as ultrahigh-performance liquid chromatography (UPLC) and gas chromatography (GC) coupled with tandem mass spectrometry (MS/MS), are widely used for the determination of pesticide residues in food ${ }^{24}$. Gas chromatography is very applicable for non-polar, volatile, and semi-volatile compounds, but not suitable for polar or ionic pesticides ${ }^{24,25}$. LC-MS/MS has become one of the most popular and effective instruments for separation, identification, and quantitation of polar and less volatile pesticides due to its high sensitivity, selectivity, and specificity when operated in multiple reaction monitoring mode ${ }^{3,25}$. Previous studies have reported the widespread use of LC-MS/MS for the determination of pesticide residues in food samples, such as honey ${ }^{26}$, hen eggs ${ }^{20}$, oregano ${ }^{27}$, green tea ${ }^{28}$, fruits and vegetables ${ }^{7,19,24,29,30}$. However, LC-MS/MS method suffers the major disadvantage that the specific masses of the compounds must be predefined before instrumental analysis, and it is limited for the identification of non-target compounds ${ }^{31,32}$. UPLC coupled to high-resolution mass spectrometry is becoming a promising strategy for multi-residue screening of pesticides $^{33}$. Quadrupole time-of-flight mass spectrometry (QTOF/MS) is a hybrid QTOF mass spectrometer with MS/MS capability, which combines the advantages of accurate mass measurement and high resolution ${ }^{25,34}$. QTOF/MS allows high sensitivity and selectivity when acquiring precursor ion and fragment information, and also allows accurate mass determination of both molecular and fragment ions, thus providing high assurance and increased efficiency for the detection, identification, and confirmation of target and non-target compounds ${ }^{25,31,32}$. Several LC-QTOF/MS methods have been reported for the analysis of pesticides, veterinary drugs, and other chemicals in food samples and drinking water ${ }^{31,32,35-37}$. However, it is still a big challenge to screen and quantify a broad range of pesticides simultaneously. The establishment of appropriate analytical methods which can overcome the difficulties such as the complexity of sample and matrix, potential interferences, large number but low concentration of compounds is urgently needed.

In this study, we developed a generic, fast, sensitive, and reliable multi-residue method for the determination of 284 pesticides, based on the pesticides listed in China's latest national food safety standard-maximum residue limits for pesticides in food (GB 2763-2019) ${ }^{18}$, using UPLC-QTOF/MS. The method was used for the analysis of 260 fruit samples collected in different districts of Shanghai to determine exposure levels and to evaluate compliance with MRLs established in Chinese and international regulations. Furthermore, the dietary risk of pesticide residues in the fruits was assessed.

\section{Results}

Method validation. A rapid method for the determination of 284 pesticides in 260 fruit samples was developed based on UPLC-QTOF/MS (Table S1). The method validation parameters, including linearity, 
equation, LOD, LOQ, recovery, and RSD, are shown in Table S2. Good linearity was observed for the analytes, with the correlation coefficients $\left(r^{2}\right)$ higher than 0.990 . The values of LOD and LOQ ranged from 0.6 to $10.0 \mu \mathrm{g} / \mathrm{kg}$ and 2.0 to $30.0 \mu \mathrm{g} / \mathrm{kg}$, respectively. The recoveries of 269 pesticides (94.7\%) ranged from $70-120 \%$, with RSDs less than $15.5 \%$ (Table S2). For remaining pesticides, 3 compounds (cyromazine, diafenthiuron, and propamocarb) showed recovery values lower than 70\% (53-69\%), and 12 compounds (bensulfuron-methyl, bifenthrin, chlorsulfuron, ethametsulfuron-methyl, ethoxysulfuron, fenpropidin, florasulam, flumetsulam, maleic hydrazide, metsulfuron-methyl, penoxsulam, and thifensulfuron-methyl) showed recovery values higher than 120\% (121-137\%), but all the RSD values did not exceed $20 \%$ (Table S2). Compared to the chromatograms of spiked sample, no chromatographic peaks close to the retention times of target analytes were found in blank samples, indicating good specificity of the method.

Pesticide residues in fruit samples. The developed method was applied to the analysis of 284 pesticides in 260 fruit samples. Matrix-matched calibration curves were used to calculate the concentrations of pesticides in fruits. Pesticide residues were detected in 228 samples ( $87.7 \%$ of the total), mainly fungicides, insecticides and acaricides. The detection rates of pesticide residues in strawberry, watermelon, melon, peach, and grape samples were $93.7 \%, 82 \%, 88 \%, 70 \%$, and $100 \%$, respectively, and more than $56 \%$ of the samples contained at least two of the analyzed pesticide residues (Fig. 1). Detailed data of the pesticide residues detected in the fruit samples are shown in Table 1. 
Table 1

Occurrence of pesticide residues in fruit samples.

\begin{tabular}{|c|c|c|c|c|c|c|c|}
\hline Fruit & Pesticide & $\begin{array}{l}\text { Min- } \\
\text { Max } \\
(\mu \mathrm{g} / \mathrm{kg})\end{array}$ & $\begin{array}{l}\text { Mean } \\
(\mu \mathrm{g} / \mathrm{kg})\end{array}$ & $\begin{array}{l}\text { China } \\
\text { MRL } \\
(\mu \mathrm{g} / \mathrm{kg})\end{array}$ & $\begin{array}{l}\text { EU } \\
\text { MRL } \\
(\mu g / k g)\end{array}$ & $\begin{array}{l}\text { No. of } \\
\text { samples } \\
>\text { China } \\
\text { MRL }\end{array}$ & $\begin{array}{l}\text { No. of } \\
\text { samples } \\
\text { > EU } \\
\text { MRL }\end{array}$ \\
\hline \multirow{14}{*}{$\begin{array}{l}\text { Strawberry } \\
(\mathrm{n}=80)\end{array}$} & Acetamiprid & $\begin{array}{l}4.6- \\
87.2\end{array}$ & 19.9 & 2000 & 500 & 0 & 0 \\
\hline & Azoxystrobin & $\begin{array}{l}4.1- \\
148.3\end{array}$ & 40.1 & 10000 & 10000 & 0 & 0 \\
\hline & Bifenazate & $\begin{array}{l}32.2- \\
540.1\end{array}$ & 151.4 & 2000 & 3000 & 0 & 0 \\
\hline & Boscalid & $\begin{array}{l}4.5- \\
1823.8\end{array}$ & 207.5 & 3000 & 6000 & 0 & 0 \\
\hline & Carbendazim & $\begin{array}{l}3.4- \\
294.8\end{array}$ & 58.5 & 500 & $100 * \star$ & 0 & $2(2.5 \%)$ \\
\hline & Chlorantraniliprole & $\begin{array}{l}8.6- \\
38.5\end{array}$ & 15.9 & $1000 *$ & 1000 & 0 & 0 \\
\hline & Cyantraniliprole & $\begin{array}{l}18.7- \\
69.6\end{array}$ & 45.5 & $4000^{*}$ & 1500 & 0 & 0 \\
\hline & Cyprodinil & $\begin{array}{l}3.8- \\
624.5\end{array}$ & 198.3 & 2000 & 5000 & 0 & 0 \\
\hline & $\begin{array}{l}\text { Diethyl } \\
\text { aminoethyl } \\
\text { hexanoate }\end{array}$ & $\begin{array}{l}20.0- \\
22.8\end{array}$ & 21.2 & - & - & - & - \\
\hline & Difenoconazole & $\begin{array}{l}5.3- \\
167.6\end{array}$ & 28.1 & - & 2000 & - & 0 \\
\hline & Dimethomorph & $\begin{array}{l}12.3- \\
33.8\end{array}$ & 23.0 & 50 & 700 & 0 & 0 \\
\hline & Dinotefuran & $\begin{array}{l}5.8- \\
44.6\end{array}$ & 25.2 & - & - & - & - \\
\hline & Ethirimol & $\begin{array}{l}5.6- \\
665.2\end{array}$ & 131.1 & - & 200 & - & $5(6.3 \%)$ \\
\hline & Etoxazole & $\begin{array}{l}4.9- \\
29.9\end{array}$ & 15.8 & - & 200 & - & 0 \\
\hline
\end{tabular}

Sum of spirotetramat and spirotetramat-enol, expressed as spirotetramat.

* Temporary MRLs prescribed by the National Standard of China.

** Lower limit of analytical determination prescribed by the EU. 


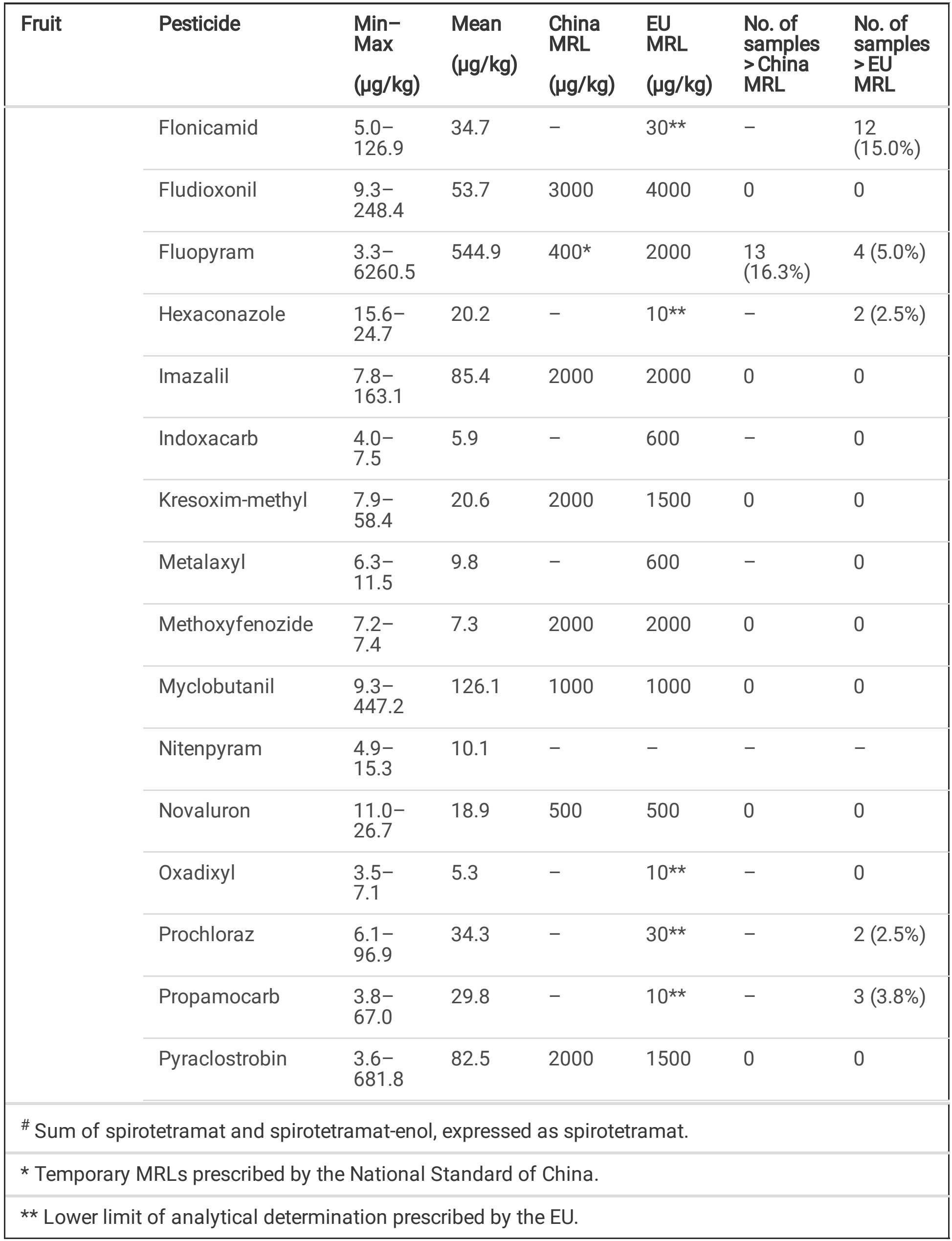




\begin{tabular}{|c|c|c|c|c|c|c|c|}
\hline Fruit & Pesticide & $\begin{array}{l}\text { Min- } \\
\text { Max } \\
(\mu g / k g)\end{array}$ & $\begin{array}{l}\text { Mean } \\
(\mu g / k g)\end{array}$ & $\begin{array}{l}\text { China } \\
\text { MRL } \\
(\mu g / k g)\end{array}$ & $\begin{array}{l}\text { EU } \\
\text { MRL } \\
(\mu g / k g)\end{array}$ & $\begin{array}{l}\text { No. of } \\
\text { samples } \\
\text { > China } \\
\text { MRL }\end{array}$ & $\begin{array}{l}\text { No. of } \\
\text { samples } \\
\text { > EU } \\
\text { MRL }\end{array}$ \\
\hline & Pyrimethanil & $\begin{array}{l}6.8- \\
2193.3\end{array}$ & 243.9 & 7000 & 5000 & 0 & 0 \\
\hline & Pyriproxyfen & 7.7 & 7.7 & - & 50 ** & - & 0 \\
\hline & Spinetoram A & $\begin{array}{l}10.8- \\
20.3\end{array}$ & 16.7 & - & 200 & - & 0 \\
\hline & Spirodiclofen & 11.9 & 11.9 & 2000 & 2000 & 0 & 0 \\
\hline & Spirotetramat ${ }^{\#}$ & $\begin{array}{l}8.3- \\
242.9\end{array}$ & 69.7 & $1500^{\star}$ & 400 & 0 & 0 \\
\hline & Sulfoxaflor & $\begin{array}{l}12.0- \\
203.0\end{array}$ & 78.3 & $500 *$ & 500 & 0 & 0 \\
\hline & Tebuconazole & $\begin{array}{l}12.0- \\
16.0\end{array}$ & 13.4 & - & $20 * \star$ & - & 0 \\
\hline & Thiacloprid & $\begin{array}{l}4.5- \\
36.5\end{array}$ & 15.9 & 1000 & 1000 & 0 & 0 \\
\hline & Thiamethoxam & $\begin{array}{l}3.8- \\
17.1\end{array}$ & 8.2 & 500 & 300 & 0 & 0 \\
\hline & $\begin{array}{l}\text { Thiophanate- } \\
\text { methyl }\end{array}$ & $\begin{array}{l}6.8- \\
1124.5\end{array}$ & 112.4 & - & $100 * \star$ & - & $2(2.5 \%)$ \\
\hline & Triadimefon & $\begin{array}{l}7.1- \\
17.0\end{array}$ & 12.0 & 700 & $10 * \star$ & 0 & $1(1.3 \%)$ \\
\hline & Triadimenol & 77.8 & 77.8 & 700 & 500 & 0 & 0 \\
\hline & Trichlorfon & 7.4 & 7.4 & 200 & $10 * \star$ & 0 & 0 \\
\hline & Trifloxystrobin & $\begin{array}{l}3.4- \\
672.4\end{array}$ & 88.3 & 1000 & 1000 & 0 & 0 \\
\hline \multirow{3}{*}{$\begin{array}{l}\text { Watermelon } \\
(n=50)\end{array}$} & Acetamiprid & $\begin{array}{l}3.6- \\
32.0\end{array}$ & 18.1 & 200 & 200 & 0 & 0 \\
\hline & Clothianidin & $\begin{array}{l}2.3- \\
13.9\end{array}$ & 7.7 & - & $20 * \star$ & - & 0 \\
\hline & Dinotefuran & $\begin{array}{l}15.3- \\
121.8\end{array}$ & 57.2 & 1000 & - & 0 & - \\
\hline
\end{tabular}

\# Sum of spirotetramat and spirotetramat-enol, expressed as spirotetramat.

* Temporary MRLs prescribed by the National Standard of China.

** Lower limit of analytical determination prescribed by the EU. 


\begin{tabular}{|c|c|c|c|c|c|c|c|}
\hline Fruit & Pesticide & $\begin{array}{l}\text { Min- } \\
\text { Max } \\
(\mu g / k g)\end{array}$ & $\begin{array}{l}\text { Mean } \\
(\mu g / k g)\end{array}$ & $\begin{array}{l}\text { China } \\
\text { MRL } \\
(\mu g / k g)\end{array}$ & $\begin{array}{l}\text { EU } \\
\text { MRL } \\
(\mu g / k g)\end{array}$ & $\begin{array}{l}\text { No. of } \\
\text { samples } \\
\text { > China } \\
\text { MRL }\end{array}$ & $\begin{array}{l}\text { No. of } \\
\text { samples } \\
>\text { EU } \\
\text { MRL }\end{array}$ \\
\hline & Ethirimol & $\begin{array}{l}2.4- \\
54.6\end{array}$ & 21.4 & - & 80 & - & 0 \\
\hline & Etoxazole & $\begin{array}{l}2.5- \\
4.4\end{array}$ & 3.5 & - & 50 & - & 0 \\
\hline & Methoxyfenozide & $\begin{array}{l}6.5- \\
15.2\end{array}$ & 9.7 & - & $10 * \star$ & - & $3(6.0 \%)$ \\
\hline & Nitenpyram & $\begin{array}{l}2.3- \\
7.2\end{array}$ & 3.5 & - & - & - & - \\
\hline & Oxadixyl & $\begin{array}{l}5.8- \\
19.2\end{array}$ & 11.5 & - & $10 \star \star$ & - & $\begin{array}{l}6 \\
(12.0 \%)\end{array}$ \\
\hline & Pymetrozine & $\begin{array}{l}3.6- \\
8.9\end{array}$ & 5.9 & - & 300 & - & 0 \\
\hline & Pyraclostrobin & 2.7 & 2.7 & 500 & 500 & 0 & 0 \\
\hline \multirow{9}{*}{$\begin{array}{l}\text { Melon } \\
(n=50)\end{array}$} & Azoxystrobin & $\begin{array}{l}2.1- \\
27.6\end{array}$ & 12.8 & - & 1000 & - & 0 \\
\hline & Difenoconazole & $\begin{array}{l}3.9- \\
12.1\end{array}$ & 6.8 & 700 & 200 & 0 & 0 \\
\hline & Ethirimol & $\begin{array}{l}7.3- \\
49.9\end{array}$ & 24.4 & - & 80 & - & 0 \\
\hline & Fluopyram & $\begin{array}{l}7.2- \\
516.8\end{array}$ & 210.9 & - & 400 & - & $3(6.0 \%)$ \\
\hline & Fosthiazate & $\begin{array}{l}3.1- \\
88.7\end{array}$ & 26.0 & - & $20 \star \star$ & - & $\begin{array}{l}11 \\
(22.0 \%)\end{array}$ \\
\hline & Indoxacarb & $\begin{array}{l}3.3- \\
6.8\end{array}$ & 4.6 & - & 500 & - & 0 \\
\hline & Paclobutrazol & $\begin{array}{l}4.0- \\
10.9\end{array}$ & 8.3 & - & $10 * \star$ & - & $1(2.0 \%)$ \\
\hline & Pyraclostrobin & $\begin{array}{l}2.6- \\
26.4\end{array}$ & 9.9 & 500 & 500 & 0 & 0 \\
\hline & Thiamethoxam & $\begin{array}{l}3.3- \\
5.9\end{array}$ & 4.4 & - & 150 & - & 0 \\
\hline
\end{tabular}

\# Sum of spirotetramat and spirotetramat-enol, expressed as spirotetramat.

* Temporary MRLs prescribed by the National Standard of China.

** Lower limit of analytical determination prescribed by the EU. 


\begin{tabular}{|c|c|c|c|c|c|c|c|}
\hline Fruit & Pesticide & $\begin{array}{l}\text { Min- } \\
\text { Max } \\
(\mu g / k g)\end{array}$ & $\begin{array}{l}\text { Mean } \\
(\mu g / k g)\end{array}$ & $\begin{array}{l}\text { China } \\
\text { MRL } \\
(\mu \mathrm{g} / \mathrm{kg})\end{array}$ & $\begin{array}{l}\text { EU } \\
\text { MRL } \\
(\mu g / k g)\end{array}$ & $\begin{array}{l}\text { No. of } \\
\text { samples } \\
\text { > China } \\
\text { MRL }\end{array}$ & $\begin{array}{l}\text { No. of } \\
\text { samples } \\
\text { > EU } \\
\text { MRL }\end{array}$ \\
\hline & Trifloxystrobin & $\begin{array}{l}3.3- \\
11.4\end{array}$ & 8.0 & - & 300 & - & 0 \\
\hline \multirow{16}{*}{$\begin{array}{l}\text { Peach } \\
(n=40)\end{array}$} & Acetamiprid & $\begin{array}{l}19.1- \\
305.2\end{array}$ & 93.0 & 2000 & 200 & 0 & $2(5.0 \%)$ \\
\hline & Carbendazim & $\begin{array}{l}5.0- \\
715.4\end{array}$ & 127.9 & 2000 & 200 & 0 & $3(7.5 \%)$ \\
\hline & Clothianidin & $\begin{array}{l}3.0- \\
77.3\end{array}$ & 19.8 & 200 & 150 & 0 & 0 \\
\hline & Cyhalothrin & $\begin{array}{l}7.2- \\
67.1\end{array}$ & 17.4 & 500 & 150 & 0 & 0 \\
\hline & Cypermethrin & $\begin{array}{l}15.8- \\
73.4\end{array}$ & 35.5 & 1000 & 2000 & 0 & 0 \\
\hline & Difenoconazole & $\begin{array}{l}3.4- \\
151.8\end{array}$ & 37.9 & 500 & 500 & 0 & 0 \\
\hline & Hexaflumuron & $\begin{array}{l}11.2- \\
122.5\end{array}$ & 39.6 & - & - & - & - \\
\hline & Imidacloprid & $\begin{array}{l}3.4- \\
157.1\end{array}$ & 43.4 & 500 & 500 & 0 & 0 \\
\hline & Indoxacarb & 4.4 & 4.4 & 1000 & 1000 & 0 & 0 \\
\hline & Nitenpyram & 4.4 & 4.4 & - & - & - & - \\
\hline & Paclobutrazol & $\begin{array}{l}4.5- \\
292.3\end{array}$ & 129.6 & - & 150 & - & $3(7.5 \%)$ \\
\hline & Pymetrozine & $\begin{array}{l}4.5- \\
14.2\end{array}$ & 7.9 & - & 30 & - & 0 \\
\hline & Pyraclostrobin & $\begin{array}{l}2.1- \\
4.7\end{array}$ & 3.2 & 1000 & 300 & 0 & 0 \\
\hline & Pyridaben & 8.1 & 8.1 & - & 300 & - & 0 \\
\hline & Pyriproxyfen & $\begin{array}{l}3.1- \\
7.8\end{array}$ & 5.4 & - & 500 & - & 0 \\
\hline & Spinosad A & 9.6 & 9.6 & $200 *$ & 600 & 0 & 0 \\
\hline
\end{tabular}

\# Sum of spirotetramat and spirotetramat-enol, expressed as spirotetramat.

* Temporary MRLs prescribed by the National Standard of China.

** Lower limit of analytical determination prescribed by the EU. 


\begin{tabular}{|c|c|c|c|c|c|c|c|}
\hline Fruit & Pesticide & $\begin{array}{l}\text { Min- } \\
\text { Max } \\
(\mu g / k g)\end{array}$ & $\begin{array}{l}\text { Mean } \\
(\mu \mathrm{g} / \mathrm{kg})\end{array}$ & $\begin{array}{l}\text { China } \\
\text { MRL } \\
(\mu g / k g)\end{array}$ & $\begin{array}{l}\text { EU } \\
\text { MRL } \\
(\mu \mathrm{g} / \mathrm{kg})\end{array}$ & $\begin{array}{l}\text { No. of } \\
\text { samples } \\
\text { > China } \\
\text { MRL }\end{array}$ & $\begin{array}{l}\text { No. of } \\
\text { samples } \\
\text { > EU } \\
\text { MRL }\end{array}$ \\
\hline & Spirodiclofen & $\begin{array}{l}12.6- \\
301.5\end{array}$ & 82.3 & 2000 & 2000 & 0 & 0 \\
\hline & $\begin{array}{l}\text { Thiophanate- } \\
\text { methyl }\end{array}$ & $\begin{array}{l}7.3- \\
52.1\end{array}$ & 19.7 & - & 2000 & - & 0 \\
\hline \multirow{7}{*}{$\begin{array}{l}\text { Grape } \\
(n=40)\end{array}$} & Difenoconazole & $\begin{array}{l}11.1- \\
408.0\end{array}$ & 106.3 & 500 & 3000 & 0 & 0 \\
\hline & Ethirimol & $\begin{array}{l}71.0- \\
677.1\end{array}$ & 309.2 & - & 500 & - & $2(5.0 \%)$ \\
\hline & Forchlorfenuron & $\begin{array}{l}2.3- \\
3.8\end{array}$ & 2.9 & 50 & $10 * \star$ & 0 & 0 \\
\hline & Methoxyfenozide & 9.9 & 9.9 & 1000 & 1000 & 0 & 0 \\
\hline & Picoxystrobin & $\begin{array}{l}4.4- \\
194.6\end{array}$ & 92.8 & 1000 & $10 * *$ & 0 & $3(7.5 \%)$ \\
\hline & Pyraclostrobin & $\begin{array}{l}31.3- \\
356.2\end{array}$ & 152.5 & 2000 & 1000 & 0 & 0 \\
\hline & Triflumuron & $\begin{array}{l}12.9- \\
1619.7\end{array}$ & 468.0 & - & $10 * *$ & - & $\begin{array}{l}40 \\
(100 \%)\end{array}$ \\
\hline \multicolumn{8}{|c|}{ \# Sum of spirotetramat and spirotetramat-enol, expressed as spirotetramat. } \\
\hline \multicolumn{8}{|c|}{ * Temporary MRLs prescribed by the National Standard of China. } \\
\hline \multicolumn{8}{|c|}{ ** Lower limit of analytical determination prescribed by the EU. } \\
\hline
\end{tabular}

A total of $44,10,10,18$, and 7 pesticides were detected in strawberry, watermelon, melon, peach, and grape samples, respectively (Table 1). The pesticides with the highest detection frequency were fluopyram $(3.3-6260.5 \mu \mathrm{g} / \mathrm{kg})$, pyraclostrobin $(3.6-681.8 \mu \mathrm{g} / \mathrm{kg})$, and flonicamid $(5.0-126.9 \mu \mathrm{g} / \mathrm{kg})$ in strawberries; dinotefuran (15.3-121.8 $\mu \mathrm{g} / \mathrm{kg})$, ethirimol $(2.4-54.6 \mu \mathrm{g} / \mathrm{kg})$, and acetamiprid $(3.6-32.0 \mu \mathrm{g} / \mathrm{kg})$ in watermelons; fosthiazate (3.1-88.7 $\mu \mathrm{g} / \mathrm{kg})$, fluopyram $(7.2-516.8 \mu \mathrm{g} / \mathrm{kg})$, and ethirimol $(7.3-49.9 \mu \mathrm{g} / \mathrm{kg})$ in melons; carbendazim (5.0-715.4 $\mu \mathrm{g} / \mathrm{kg})$, difenoconazole (3.4-151.8 $\mu \mathrm{g} / \mathrm{kg})$, and acetamiprid (19.1$305.2 \mu \mathrm{g} / \mathrm{kg}$ ) in peaches; and triflumuron (12.9-1619.7 $\mu \mathrm{g} / \mathrm{kg})$, difenoconazole $(11.1-408.0 \mu \mathrm{g} / \mathrm{kg})$, and ethirimol (71.0-677.1 $\mathrm{\mu g} / \mathrm{kg})$ in grapes (Fig. 2a-e, Table 1). The levels of fluopyram residues in 13 strawberry samples (16.3\%) were exceeded the Chinese MRL, and in 4 strawberry (5.0\%) and 3 melon samples (6.0\%) were exceeded the EU MRL (Table 1). In addition, the levels of carbendazim, ethirimol, flonicamid, hexaconazole, prochloraz, propamocarb, thiophanate-methyl, and triadimefon residues in at least one strawberry sample; methoxyfenozide and oxadixyl in at least three watermelon samples; fosthiazate and paclobutrazol in at least one melon sample; acetamiprid, carbendazim, and paclobutrazol 
in at least two peach samples; and ethirimol, picoxystrobin, and triflumuron in at least two grape samples were exceeded the EU MRLs or exceeded the lower limit of analytical determination prescribed by the EU (Table 1).

Chronic and acute dietary exposure assessment. A total of 57 pesticide residues were detected in all the fruit samples. The results of chronic and acute dietary exposure risk assessment of the pesticide residues in fruit samples are shown in Table 2 and Table 3. 
Table 2

Chronic dietary exposure assessment of detected pesticide residues in fruits among different Chinese consumer groups.

\begin{tabular}{|c|c|c|c|c|c|c|c|}
\hline \multirow[t]{2}{*}{ Pesticide } & \multirow{2}{*}{$\begin{array}{l}\text { ADI } \\
\text { (mg/kg } \\
\text { d) }\end{array}$} & \multicolumn{6}{|l|}{$\% A D I$} \\
\hline & & $\begin{array}{l}2-4 \\
\text { Male }\end{array}$ & $\begin{array}{l}2-4 \\
\text { Female }\end{array}$ & $\begin{array}{l}18-30 \\
\text { Male }\end{array}$ & $\begin{array}{l}\text { 18-30 } \\
\text { Female }\end{array}$ & $\begin{array}{l}60-70 \\
\text { Male }\end{array}$ & $\begin{array}{l}60-70 \\
\text { Female }\end{array}$ \\
\hline Acetamiprid & 0.07 & 0.5800 & 0.6201 & 0.1293 & 0.1882 & 0.1032 & 0.1199 \\
\hline Azoxystrobin & 0.2 & 0.0820 & 0.0876 & 0.0183 & 0.0266 & 0.0146 & 0.0170 \\
\hline Bifenazate & 0.01 & 4.6923 & 5.0165 & 1.0460 & 1.5226 & 0.8348 & 0.9703 \\
\hline Boscalid & 0.04 & 1.6078 & 1.7188 & 0.3584 & 0.5217 & 0.2860 & 0.3325 \\
\hline Carbendazim & 0.03 & 1.9257 & 2.0587 & 0.4293 & 0.6249 & 0.3426 & 0.3982 \\
\hline Chlorantraniliprole & 2 & 0.0025 & 0.0026 & 0.0005 & 0.0008 & 0.0004 & 0.0005 \\
\hline Clothianidin & 0.1 & 0.0852 & 0.0911 & 0.0190 & 0.0277 & 0.0152 & 0.0176 \\
\hline Cyantraniliprole & 0.03 & 0.4701 & 0.5025 & 0.1048 & 0.1525 & 0.0836 & 0.0972 \\
\hline Cyhalothrin & 0.02 & 0.2696 & 0.2883 & 0.0601 & 0.0875 & 0.0480 & 0.0558 \\
\hline Cypermethrin & 0.02 & 0.5501 & 0.5881 & 0.1226 & 0.1785 & 0.0979 & 0.1138 \\
\hline Cyprodinil & 0.03 & 2.0486 & 2.1902 & 0.4567 & 0.6648 & 0.3645 & 0.4236 \\
\hline $\begin{array}{l}\text { Diethyl aminoethyl } \\
\text { hexanoate }\end{array}$ & 0.023 & 0.2857 & 0.3054 & 0.0637 & 0.0927 & 0.0508 & 0.0591 \\
\hline Difenoconazole & 0.01 & 5.5508 & 5.9344 & 1.2374 & 1.8012 & 0.9875 & 1.1478 \\
\hline Dimethomorph & 0.2 & 0.0356 & 0.0381 & 0.0079 & 0.0116 & 0.0063 & 0.0074 \\
\hline Dinotefuran & 0.2 & 0.1277 & 0.1365 & 0.0285 & 0.0414 & 0.0227 & 0.0264 \\
\hline Ethirimol & 0.035 & 4.3045 & 4.6019 & 0.9596 & 1.3968 & 0.7658 & 0.8901 \\
\hline Etoxazole & 0.05 & 0.1196 & 0.1279 & 0.0267 & 0.0388 & 0.0213 & 0.0247 \\
\hline Flonicamid & 0.07 & 0.1536 & 0.1643 & 0.0342 & 0.0499 & 0.0273 & 0.0318 \\
\hline Fludioxonil & 0.4 & 0.0416 & 0.0445 & 0.0093 & 0.0135 & 0.0074 & 0.0086 \\
\hline Fluopyram & 0.01 & 23.4244 & 25.0429 & 5.2219 & 7.6011 & 4.1674 & 4.8438 \\
\hline Forchlorfenuron & 0.07 & 0.0128 & 0.0137 & 0.0029 & 0.0042 & 0.0023 & 0.0027 \\
\hline Fosthiazate & 0.004 & 2.0145 & 2.1537 & 0.4491 & 0.6537 & 0.3584 & 0.4166 \\
\hline Hexaconazole & 0.005 & 1.2521 & 1.3386 & 0.2791 & 0.4063 & 0.2228 & 0.2589 \\
\hline Hexaflumuron & 0.02 & 0.6137 & 0.6561 & 0.1368 & 0.1991 & 0.1092 & 0.1269 \\
\hline
\end{tabular}




\begin{tabular}{|c|c|c|c|c|c|c|c|}
\hline \multirow[t]{2}{*}{ Pesticide } & \multirow{2}{*}{$\begin{array}{l}\text { ADI } \\
\text { (mg/kg } \\
\text { d) }\end{array}$} & \multicolumn{6}{|l|}{$\% A D I$} \\
\hline & & $\begin{array}{l}2-4 \\
\text { Male }\end{array}$ & $\begin{array}{l}2-4 \\
\text { Female }\end{array}$ & $\begin{array}{l}18-30 \\
\text { Male }\end{array}$ & $\begin{array}{l}18-30 \\
\text { Female }\end{array}$ & $\begin{array}{l}60-70 \\
\text { Male }\end{array}$ & $\begin{array}{l}60-70 \\
\text { Female }\end{array}$ \\
\hline Imazalil & 0.03 & 0.8823 & 0.9432 & 0.1967 & 0.2863 & 0.1570 & 0.1824 \\
\hline Imidacloprid & 0.06 & 0.2242 & 0.2397 & 0.0500 & 0.0727 & 0.0399 & 0.0464 \\
\hline Indoxacarb & 0.01 & 0.4618 & 0.4937 & 0.1029 & 0.1498 & 0.0822 & 0.0955 \\
\hline Kresoxim-methyl & 0.4 & 0.0160 & 0.0171 & 0.0036 & 0.0052 & 0.0028 & 0.0033 \\
\hline Metalaxyl & 0.08 & 0.0380 & 0.0406 & 0.0085 & 0.0123 & 0.0068 & 0.0079 \\
\hline Methoxyfenozide & 0.1 & 0.0834 & 0.0891 & 0.0186 & 0.0271 & 0.0148 & 0.0172 \\
\hline Myclobutanil & 0.03 & 1.3027 & 1.3927 & 0.2904 & 0.4227 & 0.2318 & 0.2694 \\
\hline Nitenpyram & 0.53 & 0.0105 & 0.0113 & 0.0023 & 0.0034 & 0.0019 & 0.0022 \\
\hline Novaluron & 0.01 & 0.5858 & 0.6262 & 0.1306 & 0.1901 & 0.1042 & 0.1211 \\
\hline Oxadixyl & 0.01 & 0.5207 & 0.5567 & 0.1161 & 0.1690 & 0.0926 & 0.1077 \\
\hline Paclobutrazol & 0.1 & 0.4274 & 0.4569 & 0.0953 & 0.1387 & 0.0760 & 0.0884 \\
\hline Picoxystrobin & 0.09 & 0.3196 & 0.3417 & 0.0712 & 0.1037 & 0.0569 & 0.0661 \\
\hline Prochloraz & 0.01 & 1.0631 & 1.1365 & 0.2370 & 0.3450 & 0.1891 & 0.2198 \\
\hline Propamocarb & 0.4 & 0.0231 & 0.0247 & 0.0051 & 0.0075 & 0.0041 & 0.0048 \\
\hline Pymetrozine & 0.03 & 0.1426 & 0.1524 & 0.0318 & 0.0463 & 0.0254 & 0.0295 \\
\hline Pyraclostrobin & 0.03 & 2.5910 & 2.7700 & 0.5776 & 0.8408 & 0.4610 & 0.5358 \\
\hline Pyridaben & 0.01 & 0.2510 & 0.2684 & 0.0560 & 0.0815 & 0.0447 & 0.0519 \\
\hline Pyrimethanil & 0.2 & 0.3780 & 0.4041 & 0.0843 & 0.1226 & 0.0672 & 0.0782 \\
\hline Pyriproxyfen & 0.1 & 0.0406 & 0.0434 & 0.0091 & 0.0132 & 0.0072 & 0.0084 \\
\hline Spinetoram A & 0.05 & 0.1035 & 0.1107 & 0.0231 & 0.0336 & 0.0184 & 0.0214 \\
\hline Spinosad A & 0.02 & 0.1488 & 0.1590 & 0.0332 & 0.0483 & 0.0265 & 0.0308 \\
\hline Spirodiclofen & 0.01 & 2.9195 & 3.1213 & 0.6508 & 0.9474 & 0.5194 & 0.6037 \\
\hline Spirotetramat & 0.05 & 0.4320 & 0.4619 & 0.0963 & 0.1402 & 0.0769 & 0.0893 \\
\hline Sulfoxaflor & 0.05 & 0.4853 & 0.5189 & 0.1082 & 0.1575 & 0.0863 & 0.1004 \\
\hline Tebuconazole & 0.03 & 0.1384 & 0.1480 & 0.0309 & 0.0449 & 0.0246 & 0.0286 \\
\hline Thiacloprid & 0.01 & 0.4928 & 0.5268 & 0.1099 & 0.1599 & 0.0877 & 0.1019 \\
\hline Thiamethoxam & 0.08 & 0.0488 & 0.0522 & 0.0109 & 0.0158 & 0.0087 & 0.0101 \\
\hline
\end{tabular}




\begin{tabular}{|c|c|c|c|c|c|c|c|}
\hline \multirow[t]{2}{*}{ Pesticide } & \multirow{2}{*}{$\begin{array}{l}\text { ADI } \\
(\mathrm{mg} / \mathrm{kg} \\
\text { d) }\end{array}$} & \multicolumn{6}{|l|}{$\% A D I$} \\
\hline & & $\begin{array}{l}2-4 \\
\text { Male }\end{array}$ & $\begin{array}{l}2-4 \\
\text { Female }\end{array}$ & $\begin{array}{l}18-30 \\
\text { Male }\end{array}$ & $\begin{array}{l}18-30 \\
\text { Female }\end{array}$ & $\begin{array}{l}60-70 \\
\text { Male }\end{array}$ & $\begin{array}{l}60-70 \\
\text { Female }\end{array}$ \\
\hline Thiophanate-methyl & 0.09 & 0.4549 & 0.4863 & 0.1014 & 0.1476 & 0.0809 & 0.0941 \\
\hline Triadimefon & 0.03 & 0.1240 & 0.1325 & 0.0276 & 0.0402 & 0.0221 & 0.0256 \\
\hline Triadimenol & 0.03 & 0.8037 & 0.8593 & 0.1792 & 0.2608 & 0.1430 & 0.1662 \\
\hline Trichlorfon & 0.002 & 1.1467 & 1.2260 & 0.2556 & 0.3721 & 0.2040 & 0.2371 \\
\hline Trifloxystrobin & 0.04 & 0.7462 & 0.7977 & 0.1663 & 0.2421 & 0.1327 & 0.1543 \\
\hline Triflumuron & 0.014 & 10.3605 & 11.0763 & 2.3096 & 3.3619 & 1.8432 & 2.1424 \\
\hline
\end{tabular}


Table 3

Acute dietary exposure assessment of detected pesticide residues in fruits among different Chinese consumer groups.

\begin{tabular}{|c|c|c|c|c|c|c|c|}
\hline \multirow[t]{2}{*}{ Pesticide } & \multirow{2}{*}{$\begin{array}{l}\text { ARfD } \\
\text { (mg/kg } \\
\text { d) }\end{array}$} & \multicolumn{6}{|l|}{ \%ARfD } \\
\hline & & $\begin{array}{l}2-4 \\
\text { Male }\end{array}$ & $\begin{array}{l}2-4 \\
\text { Female }\end{array}$ & $\begin{array}{l}18-30 \\
\text { Male }\end{array}$ & $\begin{array}{l}\text { 18-30 } \\
\text { Female }\end{array}$ & $\begin{array}{l}60-70 \\
\text { Male }\end{array}$ & $\begin{array}{l}60-70 \\
\text { Female }\end{array}$ \\
\hline Acetamiprid & 0.1 & 10.2155 & 10.7491 & 3.5789 & 4.1164 & 3.5322 & 3.9876 \\
\hline Carbendazim & 0.5 & 4.8633 & 5.1173 & 1.7038 & 1.9597 & 1.6816 & 1.8983 \\
\hline Clothianidin & 0.6 & 0.3658 & 0.3849 & 0.1282 & 0.1474 & 0.1265 & 0.1428 \\
\hline Cyhalothrin & 0.02 & 8.0740 & 8.4958 & 2.8287 & 3.2535 & 2.7917 & 3.1516 \\
\hline Cypermethrin & 0.04 & 4.4148 & 4.6454 & 1.5467 & 1.7790 & 1.5265 & 1.7233 \\
\hline Difenoconazole & 0.3 & 5.9327 & 6.2426 & 2.0785 & 2.3906 & 2.0513 & 2.3158 \\
\hline Dimethomorph & 0.6 & 0.1356 & 0.1427 & 0.0475 & 0.0546 & 0.0469 & 0.0529 \\
\hline Dinotefuran & 1 & 0.4005 & 0.4214 & 0.1403 & 0.1614 & 0.1385 & 0.1563 \\
\hline Fluopyram & 0.5 & 32.6272 & 34.3316 & 11.4307 & 13.1474 & 11.2815 & 12.7358 \\
\hline Imazalil & 0.05 & 7.8510 & 8.2611 & 2.7505 & 3.1636 & 2.7146 & 3.0646 \\
\hline Imidacloprid & 0.4 & 0.9454 & 0.9947 & 0.3312 & 0.3809 & 0.3269 & 0.3690 \\
\hline Indoxacarb & 0.1 & 0.4492 & 0.4726 & 0.1574 & 0.1810 & 0.1553 & 0.1753 \\
\hline Methoxyfenozide & 0.9 & 0.0870 & 0.0915 & 0.0305 & 0.0351 & 0.0301 & 0.0340 \\
\hline Picoxystrobin & 0.09 & 5.2043 & 5.4762 & 1.8233 & 2.0971 & 1.7995 & 2.0315 \\
\hline Prochloraz & 0.1 & 2.3315 & 2.4533 & 0.8168 & 0.9395 & 0.8062 & 0.9101 \\
\hline Propamocarb & 2 & 0.0806 & 0.0849 & 0.0283 & 0.0325 & 0.0279 & 0.0315 \\
\hline Pymetrozine & 0.1 & 0.5565 & 0.5856 & 0.1950 & 0.2243 & 0.1924 & 0.2172 \\
\hline Pyraclostrobin & 0.05 & 51.6005 & 54.2960 & 18.0778 & 20.7929 & 17.8419 & 20.1420 \\
\hline Spirotetramat & 1 & 0.5846 & 0.6151 & 0.2048 & 0.2356 & 0.2021 & 0.2282 \\
\hline Sulfoxaflor & 0.3 & 1.6290 & 1.7141 & 0.5707 & 0.6564 & 0.5632 & 0.6359 \\
\hline Tebuconazole & 0.3 & 0.1282 & 0.1349 & 0.0449 & 0.0517 & 0.0443 & 0.0500 \\
\hline Thiacloprid & 0.03 & 2.9286 & 3.0816 & 1.0260 & 1.1801 & 1.0126 & 1.1432 \\
\hline Thiamethoxam & 1 & 0.0553 & 0.0582 & 0.0194 & 0.0223 & 0.0191 & 0.0216 \\
\hline Triadimefon & 0.08 & 0.5106 & 0.5373 & 0.1789 & 0.2058 & 0.1766 & 0.1993 \\
\hline Triadimenol & 0.08 & 2.3409 & 2.4632 & 0.8201 & 0.9433 & 0.8094 & 0.9138 \\
\hline
\end{tabular}


For the chronic dietary exposure assessment, ADI values were obtained from the National Standard of China ${ }^{18}$. In different age and sex groups, the risk of chronic dietary intake (\%ADI) of the 57 pesticides from eating the fruits was less than $100 \%$ (Table 2). The risk values of fluopyram and triflumuron were the highest, especially in the 2-4 year old children groups (\%ADI > 10\%), followed by difenoconazole, bifenazate, and ethirimol (Table 2). Among the different Chinese population groups, the risk of chronic dietary intake of the pesticides was in the following order: 2-4 year old female children $>2-4$ year old male children $>18-30$ year old female adult $>18-30$ year old male adult $>60-70$ year old female adult $>$ 60-70 year old male adult.

For the acute dietary exposure assessment, ARfD values were obtained from the joint Food and Agriculture Organization of the United Nations (FAO) and World Health Organization (WHO) Meeting on Pesticide Residues (JMPR) ${ }^{38}$. A list of pesticides has been evaluated by the JMPR, and it has decided that ARfD values were unnecessary for 21 pesticides, including azoxystrobin, bifenazate, boscalid, chlorantraniliprole, cyantraniliprole, cyprodinil, etoxazole, flonicamid, fludioxonil, fosthiazate, kresoximmethyl, metalaxyl, myclobutanil, novaluron, pyrimethanil, pyriproxyfen, spinetoram A, spinosad A, spirodiclofen, thiophanate-methyl, and trifloxystrobin ${ }^{38}$. In addition, there are no regulations for ARfD values for diethyl aminoethyl hexanoate, ethirimol, forchlorfenuron, hexaconazole, hexaflumuron, nitenpyram, oxadixyl, paclobutrazol, pyridaben, trichlorfon, and triflumuron so far. Thus, the risk values of acute dietary exposure (\%ARfD) of 25 pesticides are given in Table 3 . All the \%ARfD values were lower than $100 \%$ in all the consumption groups. The risk of acute intake in different groups was in the order of 2-4 year old female children $>2-4$ year old male children $>18-30$ year old female adult $>60-70$ year old female adult $>18-30$ year old male adult $>60-70$ year old male adult. Pyraclostrobin, fluopyram, and acetamiprid exhibited higher acute risks for Chinese consumers than other pesticides, their \%ARfD values were $17.8419-54.2960 \%, 11.2815-34.3316 \%$, and $3.5322-10.7491 \%$, respectively.

\section{Discussion}

The linearity (>0.990) was considered acceptable ${ }^{39}$. Most of the LOQ values $(82.4 \%)$ were below the nondetectable default value $(0.01 \mathrm{mg} / \mathrm{kg})$ recommended in the EU regulations ${ }^{16}$. The obtained LOQs were much lower than the Chinese and EU MRLs $(0.01-10 \mathrm{mg} / \mathrm{kg})$ for pesticides in strawberries, watermelons, melons, peaches, and grapes ${ }^{18,22}$, indicating that the developed method is sensitive and suitable for comprehensive monitoring of pesticide residues in the fruit samples. The SANTE guidelines recommend that the acceptable mean recoveries are those within the range $70-120 \%$, with an associated RSD $\leq$ $20 \%{ }^{40}$. The accuracy and precision obtained in this study are comparable with those reported in previous studies. Yang et al. ${ }^{32}$ determined 50 pesticides in starfruit and Indian jujube using LC-QTOF/MS, and obtained recoveries between $63 \%$ and $119 \%$, with RSDs of $0.2-3.2 \%$. Sivaperumal et al. ${ }^{31}$ achieved satisfactory recoveries ranging from $74-111 \%$, with RSDs below $13.2 \%$. 
Multiple pesticide residues in fruits are commonly observed. Li et al. ${ }^{41}$ found that carbendazim, cyhalothrin, acetamiprid, cypermethrin, imidacloprid, as well as difenoconazole had high detection frequency in peaches. Previous studies have also noted that pesticides, especially fungicides and insecticides such as carbendazim, pyrimethanil, trifloxystrobin, and acetamiprid, had high detection frequency in strawberry fruits in China, Poland, and Turkey $1,30,42$. In this study, the strawberry samples were collected in January when strawberries first appeared on market. In order to increase strawberry yield and maximize returns, growers apply high levels of various pesticides during this period. Chu et al. ${ }^{30}$ also noted that the detection rates of pesticides in strawberries collected in January were higher than that in strawberries collected in other months.

MRLs are the maximum permissible values of pesticide residues in food. They are established to ensure the proper use of pesticides in agriculture and reduce harmful pesticide intake in humans, and thus protect human health ${ }^{17}$. The overall result revealed that $95.0 \%$ of samples were below the MRLs prescribed by the National Standard of China ${ }^{18}$, and $66.2 \%$ of samples were below the MRLs prescribed by the $\mathrm{EU}^{16}$. Currently, there are many pesticides which are registered for use on fruits in China (China Pesticide Information Network. http://www.icama.org.cn/hysj/index.jhtml). In this study, only 11, 3, 1, 5, and 4 detected pesticides are registered pesticides in strawberries, watermelons, melons, peaches, and grapes, respectively. Moreover, some pesticides had no corresponding residue limits authorized in Chinese regulations but had high detection rates, such as ethirimol in strawberries, watermelons, melons, and grapes, fosthiazate and fluopyram in melons, and triflumuron in grapes. This is a great challenge for government to monitor the use of pesticides.

The results of chronic and acute dietary exposure assessment are in agreement with the results of Chu et al. ${ }^{30}$. Similar results from studies on chronic dietary risk of pesticides in fruits in Poland and in peaches in China have also been reported ${ }^{1,41}$. They also indicated that the chronic risk values for children were higher than that for adults, but neither exceeded $100 \%$. Through fruit consumption, children had higher chronic and acute exposure risks than adults, and females had higher exposure risks than males, which supports previous findings $30,43,44$. In our study, the evaluated fruits exhibited an acceptably low risk to Chinese consumers, however, other studies indicated that some kind of pesticide residues in fruits showed unacceptable acute risks, especially for infants and children ${ }^{41,43,44}$. Although pesticides with high detection rates do not means high exposure risks, the potential risks should be paid more attention to the pesticides with high levels (>MRLs) and the pesticides with no regulations for ARfD values. For example, ethirimol showed relatively high detection rates in the fruits, and the levels exceeded the EU MRLs in some strawberry and grape samples, and triflumuron was detected in all the grape samples and showed relatively high chronic dietary risk, but we could not evaluate their acute dietary risks. Such a risk cannot be excluded, especially for children and females.

In order to decrease the MRL-exceeding rate of pesticide residues in fruits, certain measures, such as increased education of growers, control of the sale and use of pesticides, rigorous monitoring of pesticides before harvest, implementation of integrated pest management methods, as well as 
improvement of regulations, are urgently needed ${ }^{17,42}$. Both growers and government are responsible for food safety, the applications and monitoring programs for pesticides in domestic products must be responsibly carried out.

\section{Materials And Methods}

Chemicals and reagents. High-performance liquid chromatography (HPLC) grade methanol and acetonitrile were obtained from Merck (Darmstadt, Germany), ammonium acetate $\left(\mathrm{CH}_{3} \mathrm{COONH}_{4}\right)$ was purchased from ANPEL Laboratory Technologies Inc. (Shanghai, China). Analytical grade sodium chloride $(\mathrm{NaCl})$ was purchased from Shanghai Titan Scientific Co., Ltd. (Shanghai, China). Ultrapure water was prepared using a Milli-Q purification system (Millipore, Billerica, MA, USA).

Certified pesticide standard solutions ( $100 \mu \mathrm{g} / \mathrm{mL}$ in HPLC-grade acetonitrile, methanol, and acetone) were purchased from A ChemTek, Inc. (Worcester, MA, USA). These stock solutions were stored at $-20{ }^{\circ} \mathrm{C}$ in the dark. From each stock solution, a mixed standard working solution contained all the pesticides was prepared at $1 \mu \mathrm{g} / \mathrm{mL}$ by appropriate dilution with acetonitrile/Milli-Q water $(1 / 1, \mathrm{v} / \mathrm{v})$, which was stored at $4{ }^{\circ} \mathrm{C}$ and renewed every 2 months.

Sample preparation. A total of 260 fruit samples, including five local cultivars: strawberry (Fragaria $\times$ ananassa Duch.), watermelon (Citrullus lanatus (Thunb.) Mansfeld), melon (Cucumis melo L.), peach (Amygdalus persica L.), and grape (Vitis vinifera L.), were collected from different fields in different districts of Shanghai, China (Table S3) in 2019 and 2020. All the fruit samples were collected with the permission of the farmers. The wet weight for one sample was $3 \mathrm{~kg}$ for strawberry, melon, peach, grape, and tomato, and each watermelon sample include three watermelons. All samples were kept cool and edible portions of the analytical samples were homogenized in a commercial blender, and then stored at $20^{\circ} \mathrm{C}$ in the dark until chemical analysis.

Pesticides in fruit samples were extracted using a modified method of Yang et al. ${ }^{32}$. A blended sample (around $10 \mathrm{~g}$ ) was weighed into a $50 \mathrm{~mL}$ polypropylene centrifuge tube, and mixed thoroughly with $10 \mathrm{~mL}$ acetonitrile for 20 min using an advanced multi-tube vortexer (Troemner LLC., Thorofare, NJ, USA). Subsequently, $5 \mathrm{~g}$ of $\mathrm{NaCl}$ was added to the tube and vortexed for $1 \mathrm{~min}$. The mixture was centrifuged at $5000 \mathrm{rpm}$ for $5 \mathrm{~min}$ in a Thermo Fisher ST 16R centrifuge (Osterode, Germany). After centrifugation, $1 \mathrm{~mL}$ of the supernatant was transferred to a $10 \mathrm{~mL}$ glass tube, $1 \mathrm{~mL}$ of Milli-Q water was added, and the tube was vortexed for $30 \mathrm{~s}$. The extract was then filtered through a $0.22 \mu \mathrm{m}$ organic syringe filter (Millipore, Bedford, MA, USA) and $3 \mu \mathrm{L}$ were injected into the UPLC-QTOF/MS system.

UPLC-QTOF/MS analysis. The pesticides were identified and quantified using an ultrahigh-performance liquid chromatography system (Waters Acquity I-Class, Waters Corporation, Milford, MA, USA) coupled to a quadrupole time-of-fight mass spectrometer (AB SCIEX TripleTOF ${ }^{\text {TM }} 5600+$, Framingham, MA, USA). The chromatographic conditions, including the selection of chromatographic column, mobile phase and buffer solution, and the gradient elution program, were optimized to achieve good separation. The chromatographic separation was performed on a Waters Acquity UPLC $®$ HSS T3 column $(2.1 \times 100$ mm; 
particle size $1.8 \mu \mathrm{m}$, Waters, Ireland) with a flow rate of $0.4 \mathrm{~mL} / \mathrm{min}$, and the column temperature was kept at $45{ }^{\circ} \mathrm{C}$. Mobile phases were $100 \%$ methanol (solvent A) and $5 \mathrm{mM}$ ammonium acetate in Milli-Q water (solvent B). The gradient for solvent A was as follows: 0-0.5 min, 2\%; 0.5-15 min, 2-98\%; 15-17 min, $98 \%$; $17-17.1 \mathrm{~min}, 98-2 \%$; $17.1-20 \mathrm{~min}, 2 \%$. The injection volume was $3 \mu \mathrm{L}$.

The QTOF/MS spectra were acquired in positive electrospray ionization mode $\left(\mathrm{ESI}^{+}\right)$with the following parameters: mass range, $50-1000 \mathrm{~m} / \mathrm{z}$, ionspray voltage floating (ISVF), $5500 \mathrm{~V}$; temperature (TEM), 500 ${ }^{\circ} \mathrm{C}$; ion source gas (GS1) nebulizer gas pressure, $50 \mathrm{psi}$; ion source gas (GS2) auxiliary heater gas pressure, 50 psi; curtain gas (CUR), 35 psi; declustering potential (DP), 80 V; collision energy (CE), $35 \pm 15$ $\mathrm{eV}$. The mass spectrometry analysis was conducted in full scan TOF/MS mode and in MS/MS mode. Detailed instrument conditions are described in Yang et al. ${ }^{32}$.

Method validation. The fruit samples were analyzed by UPLC-QTOF/MS in advance, and the sample detected as pesticide-free was used as the blank matrix sample for the spiking experiment. The validation parameters included linearity, sensitivity, accuracy, precision, and specificity. The linearity was determined using matrix-matched calibration curves, which were obtained by adding mixed pesticide standard solution into the extract of blank matrix at seven concentration levels in the range of $2-200 \mu \mathrm{g} / \mathrm{L}$, analyzed in triplicate. The sensitivity was assessed by limits of detection (LODs) and limits of quantitation (LOQs). The values of LODs and LOQs were determined by injecting spiked blank sample and calculated as the lowest amount according to signal-to-noise $(\mathrm{S} / \mathrm{N})$ ratios of 3 and 10 , respectively. Method accuracy was evaluated by recovery studies. The blank matrix sample was spiked at two concentration levels ( 10 and $100 \mu \mathrm{g} / \mathrm{kg}$ ) with six replicates for each level, then the spiked samples were extracted according to the procedure as described in Section Sample preparation. The relative standard deviation (RSD) of the pesticides from the recovery studies were used to evaluate the precision. To assess the specificity, the chromatograms of blank sample and spiked sample at LOD levels were analyzed. The $\mathrm{S} / \mathrm{N}$ ratios of chromatographic peaks in blank sample had to be lower than that in spiked sample ${ }^{39}$.

Dietary exposure risk assessment. The chronic and acute dietary exposure risk values were determined by comparing the value of national estimated daily intake (NEDI) of pesticides with acceptable daily intake (ADI), and by comparing the value of estimated short-term intake (ESTI) of pesticides with acute reference dose (ARfD), respectively, according to the following equations $30,45,46$.

$\mathrm{NEDI}=\left(\sum \mathrm{R} \times \mathrm{F}\right) / \mathrm{bw}(1)$

$\% A D I=(N E D I / A D I) \times 100 \%(2)$

The chronic risk was calculated using the above equations (1) and (2). NEDI (mg/kg.d) indicates the national estimated daily intake; $R(\mathrm{mg} / \mathrm{kg})$ is the mean amount of pesticide residues in fruit samples; $F$ $(\mathrm{kg} / \mathrm{d})$ is the dietary consumption of fruits in China; bw $(\mathrm{kg})$ is the average body weight; $A D I(\mathrm{mg} / \mathrm{kg} \cdot \mathrm{d})$ is the acceptable daily intake.

$\mathrm{ESTI}=\left(\sum \mathrm{HR} \times \mathrm{LP}\right) / \mathrm{bw}(3)$ 
$\% A R f D=(E S T I / A R f D) \times 100 \%(4)$

The acute risk was calculated using the above equations (3) and (4). ESTI ( $\mathrm{mg} / \mathrm{kg} \cdot \mathrm{d})$ represents the estimated short-term intake; $\mathrm{HR}(\mathrm{mg} / \mathrm{kg})$ is the highest amount of pesticide residues in fruit samples; LP $(\mathrm{kg} / \mathrm{d})$ is the large portion of fruit consumption in Chinese population; ARfD $(\mathrm{mg} / \mathrm{kg} \cdot \mathrm{d})$ is the acute reference dose.

In this study, fruit consumption group was divided into three sensitive population groups, including 2-4, 18-30, and 60-70 year old male and female groups. The average body weight and fruit consumption in different groups in China are shown in Table S4. If \%ADI or \%ARfD value is lower than $100 \%$, the exposure risk is acceptable. The higher the value, the greater the risk. While when the value is higher than $100 \%$, it indicates an unacceptably high risk to consumers ${ }^{30,45}$.

\section{Declarations}

\section{Data availability}

All data generated and/or analyzed during the current study are available from the corresponding author on reasonable request.

\section{Acknowledgements}

This work was supported by the Shanghai Agriculture Applied Technology Development Program, China (2019-02-08-00-12-F01144) and China Postdoctoral Science Foundation (2019M661582).

\section{Author Contributions}

Y.Z. and L.C. performed the experimental. Y.Z. and W.S. developed and validated the method and analyzed the data. Y.Z. and G.S. evaluated the result and prepared the manuscript. B.B. and C.Z. reviewed and edited the manuscript. All authors revised the manuscript and approved the final form.

\section{Additional Information}

\section{Supplementary Information}

Competing Interests: The authors declare no conflict of interest.

Approvals and Permissions: This study was approved by Shanghai Municipal Agriculture and Rural Affairs Committee (Approval number: 2019-02-08-00-12-F01144). The experiment was performed in accordance with the regulations (NY/T 789-2004) established by the Ministry of Agriculture and Rural Affairs of the People's Republic of China. All the farms or farmer professional cooperatives are legally registered in Shanghai.

\section{References}


1. Szpyrka, E. et al. Evaluation of pesticide residues in fruits and vegetables from the region of southeastern Poland. Food Control. 48, 137-142 (2015).

2. Méndez-Lagunas, L., Rodríguez-Ramírez, J., Cruz-Gracida, M., Sandoval-Torres, S. \& Barriada-Bernal, G. Convective drying kinetics of strawberry (Fragaria ananassa): effects on antioxidant activity, anthocyanins and total phenolic content. Food Chem. 230, 174-181 (2017).

3. Oshita, D. \& Jardim, I. C. S. F. Comparison of different sorbents in the QuEChERS method for the determination of pesticide residues in strawberries by LC-MS/MS. Chromatographia. 77, 1291-1298 (2014).

4. Sun, H. et al. Residue analysis and dietary exposure risk assessment of acibenzolar-S-methyl and its metabolite acibenzolar acid in potato, garlic, cabbage, grape and tomato. Ecotoxicol. Environ. Saf. 207, 111178 (2021).

5. Saber, A. N., Malhat, F. M., Badawy, H. M. A. \& Barakat, D. A. Dissipation dynamic, residue distribution and processing factor of hexythiazox in strawberry fruits under open field condition. Food Chem. 196, 1108-1116 (2016).

6. Wang, Z. et al. Screening for suitable chemical acaricides against two-spotted spider mites, Tetranychus urticae, on greenhouse strawberries in China. Ecotoxicol. Environ. Saf. 163, 63-68 (2018).

7. Grimalt, S. \& Dehouck, P. Review of analytical methods for the determination of pesticide residues in grapes. J. Chromatogr. A. 1433, 1-23 (2016).

8. Environmental Working Group (EWG)'s 2020 Shopper's Guide to Pesticides in Produce ${ }^{\text {TM }}$. March 25, 2020. Available at https://www.ewg.org/foodnews/summary.php (accessed July 2020).

9. Lorenz, J. G., Costa, L. L. F., Suchara, E. A. \& Sant'Anna, E. S. Multivariate optimization of the QuEChERS-GC-ECD method and pesticide investigation residues in apples, strawberries, and tomatoes produced in Brazilian south. J. Braz. Chem. Soc. 25, 1583-1591 (2014).

10. Querejeta, G. A. et al. Environmental pesticide distribution in horticultural and floricultural periurban production units. Chemosphere. 87, 566-572 (2012).

11. Abd-Elhaleem, Z. A. Pesticide residues in tomato and tomato products marketed in Majmaah province, KSA, and their impact on human health. Environ. Sci. Pollut. Res. 27, 8526-8534 (2020).

12. Alavanja, M. C. R., Ross, M. K. \& Bonner, M. R. Increased cancer burden among pesticide applicators and others due to pesticide exposure. CA Cancer J. Clin. 63, 120-142 (2013).

13. Markel, T. A., Proctor, C., Ying, J. \& Winchester, P. D. Environmental pesticides increase the risk of developing hypertrophic pyloric stenosis. J. Pediatr. Surg. 50, 1283-1288 (2015).

14. Houbraken, M., Bauweraerts, I., Fevery, D., Van Labeke, M. C. \& Spanoghe, P. Pesticide knowledge and practice among horticultural workers in the Lâm Đồng region, Vietnam: a case study of chrysanthemum and strawberries. Sci. Total Environ. 550, 1001-1009 (2016).

15. Sharma, A. et al. Global trends in pesticides: a looming threat and viable alternatives. Ecotoxicol. Environ. Saf. 201, 110812 (2020). 
16. Commission Regulation (EC) 396/2005 of the European parliament and of the council of 23 February 2005 on maximum residue levels of pesticides in or on food and feed of plant and animal origin and amending council directive 91/414/EEC. 2005R0396-EN-13.05.2016-019.001, 1-2640.

17. Concha-meyer, A. et al. Pesticide residues quantification in frozen fruit and vegetables in Chilean domestic market using QuEChERS extraction with ultra-high-performance liquid chromatography electrospray ionization Orbitrap mass spectrometry. Food Chem. 295, 64-71 (2019).

18. China National Standard GB 2763-2019: National food safety standard-Maximum residue limits for pesticides in food. Beijing (China): National Health Commission, Ministry of Agriculture and Rural Affairs, and State Administration for Market Regulation of the People's Republic of China.

19. Silva, R. D. O. et al. Efficiency of ESI and APCI ionization sources in LC-MS/MS systems for analysis of 22 pesticide residues in food matrix. Food Chem. 297, 124934 (2019).

20. Song, N. E. et al. Determination of 60 pesticides in hen eggs using the QuEChERS procedure followed by LC-MS/MS and GC-MS/MS. Food Chem. 298, 125050 (2019).

21. Kin, C. M. \& Huat, T. G. Headspace solid-phase microextraction for the evaluation of pesticide residue contents in cucumber and strawberry after washing treatment. Food Chem. 123, 760-764 (2010).

22. EU-Database. EU Pesticides Database (search pesticide residues). Available at https://ec.europa.eu/food/plant/pesticides/eu-pesticides-database/public/? event=pesticide.residue.selection\&language=EN (accessed Dec 2020).

23. Lozano, A. et al. Miniaturisation and optimisation of the Dutch mini-Luke extraction method for implementation in the routine multi-residue analysis of pesticides in fruits and vegetables. Food Chem. 192, 668-681 (2016).

24. Muñoz, N. C. et al. Determination of pesticide residues in golden berry (Physalis peruviana L.) by modified QuEChERS method and ultra-high performance liquid chromatography-tandem quadrupole mass spectrometry. Food Anal. Methods. 10, 320-329 (2017).

25. Wang, X., Wang, S. \& Cai, Z. The latest developments and applications of mass spectrometry in foodsafety and quality analysis. Trends Anal. Chem. 52, 170-185 (2013).

26. Gawet, M. et al. Determination of neonicotinoids and 199 other pesticide residues in honey by liquid and gas chromatography coupled with tandem mass spectrometry. Food Chem. 282, 36-47 (2019).

27. Drabova, L. et al. Food fraud in oregano: pesticide residues as adulteration markers. Food Chem. 276, 726-734 (2019).

28. Huang, Y. et al. Determination of multi-pesticide residues in green tea with a modified QuEChERS protocol coupled to HPLC-MS/MS. Food Chem. 275, 255-264 (2019).

29. Yang, X., Luo, J., Li, S. \& Liu, C. Evaluation of nine pesticide residues in three minor tropical fruits from southern China. Food Control. 60, 677-682 (2016).

30. Chu, Y. et al. Simultaneous determination of 98 pesticide residues in strawberries using UPLC-MS/MS and GC-MS/MS. Microchem. J. 156, 104975 (2020).

31. Sivaperumal, P., Anand, P. \& Riddhi, L. Rapid determination of pesticide residues in fruits and vegetables, using ultra-high-performance liquid chromatography/time-of-flight mass spectrometry. 
Food Chem. 168, 356-365 (2015).

32. Yang, X., Luo, J., Duan, Y., Li, S. \& Liu, C. Simultaneous analysis of multiple pesticide residues in minor fruits by ultrahigh-performance liquid chromatography/hybrid quadrupole time-of-fight mass spectrometry. Food Chem. 241, 188-198 (2018).

33. Wang, S., Kong, C., Chen, Q. \& Yu, H. Screening 89 pesticides in fishery drugs by ultrahigh performance liquid chromatography tandem quadrupole-orbitrap mass spectrometer. Molecules. 24, 3375 (2019).

34. Muehlwald, S., Buchner, N. \& Kroh, L. W. Investigating the causes of low detectability of pesticides in fruits and vegetables analysed by high-performance liquid chromatography - Time-of-flight. J. Chromatogr. A. 1542, 37-49 (2018).

35. Zhang, Y. et al. Rapid screening and quantification of multi-class multi-residue veterinary drugs in royal jelly by ultra performance liquid chromatography coupled to quadrupole time-of-flight mass spectrometry. Food Control. 60, 667-676 (2016).

36. Tak, V. et al. Simultaneous detection and identification of precursors, degradation and co-products of chemical warfare agents in drinking water by ultra-high performance liquid chromatographyquadrupole time-of-flight mass spectrometry. J. Chromatogr. A. 1370, 80-92 (2014).

37. Wang, H. X., Zhou, Y. \& Jiang, Q. W. Simultaneous screening of estrogens, progestogens, and phenols and their metabolites in potable water and river water by ultra-performance liquid chromatography coupled with quadrupole time-of-flight mass spectrometry. Microchem. J. 100, 83-94 (2012).

38. The Joint FAO/WHO Meeting on Pesticide Residues (JMPR). Available at http://www.fao.org/agriculture/crops/thematic-sitemap/theme/pests/Ipe/Ipe-c/en/ (accessed Dec 2020).

39. Zhao, Z. et al. Ion-exchange solid-phase extraction combined with liquid chromatography-tandem mass spectrometry for the determination of veterinary drugs in organic fertilizers. J. Chromatogr. $B$. 1022, 281-289 (2016).

40. SANTE. European Commission. Guidance document on analytical quality control and method validation procedures for pesticide residues analysis in food and feed. Document No. SANTE/12682/2019,1-48.

41. Li, Z. et al. A monitoring survey and dietary risk assessment for pesticide residues on peaches in China. Regul. Toxicol. Pharmacol. 97, 152-162 (2018).

42. Bakırcı, G. T., Acay, Y., Bakırcı, D. B., Ötleş, S. \& F. \& Pesticide residues in fruits and vegetables from the Aegean region, Turkey. Food Chem. 160, 379-392 (2014).

43. Lozowicka, B. et al. Toxicological studies for adults and children of insecticide residues with common mode of action (MoA) in pome, stone, berries and other small fruit. Sci. Total Environ. 566-567, 144156 (2016).

44. Mojsak, P., Łozowicka, B. \& Kaczyński, P. Estimating acute and chronic exposure of children and adults to chlorpyrifos in fruit and vegetables based on the new, lower toxicology data. Ecotoxicol. Environ. Saf. 159, 182-189 (2018). 
45. Chen, X., Fan, X., Ma, Y. \& Hu, J. Dissipation behaviour, residue distribution and dietary risk assessment of tetraconazole and kresoxim-methyl in greenhouse strawberry via RRLC-QqQ-MS/MS technique. Ecotoxicol. Environ. Saf. 148, 799-804 (2018).

46. Heshmati, A., Mehri, F. \& Khaneghah, A. M. Simultaneous multi-determination of pesticide residues in black tea leaves and infusion: a risk assessment study. Environ. Sci. Pollut. Res. [Online early access] https://doi.org/10.1007/s11356-020-11658-3 (2020).

\section{Figures}

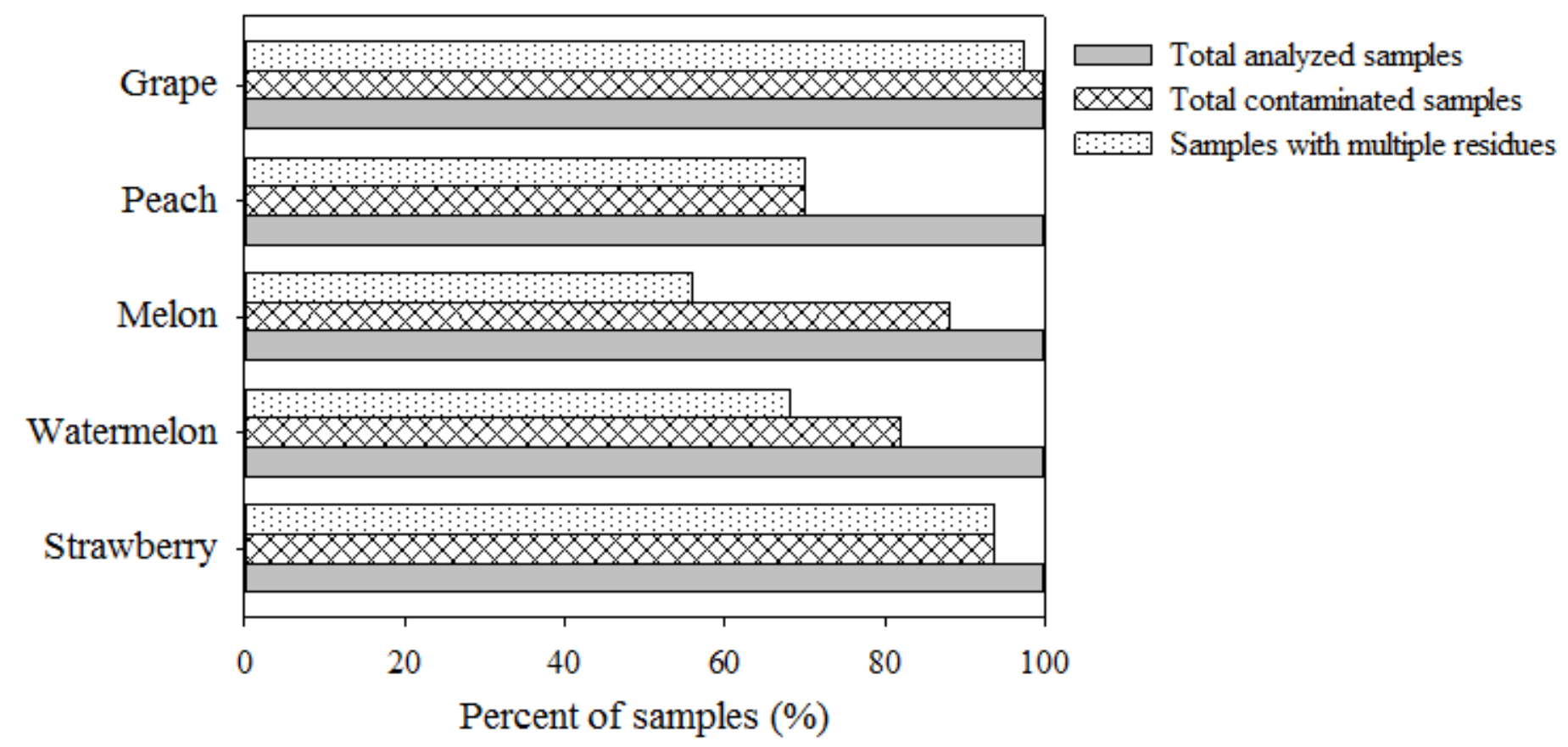

\section{Figure 1}

Percent of contaminated samples in strawberries, watermelons, melons, peaches, and grapes. 


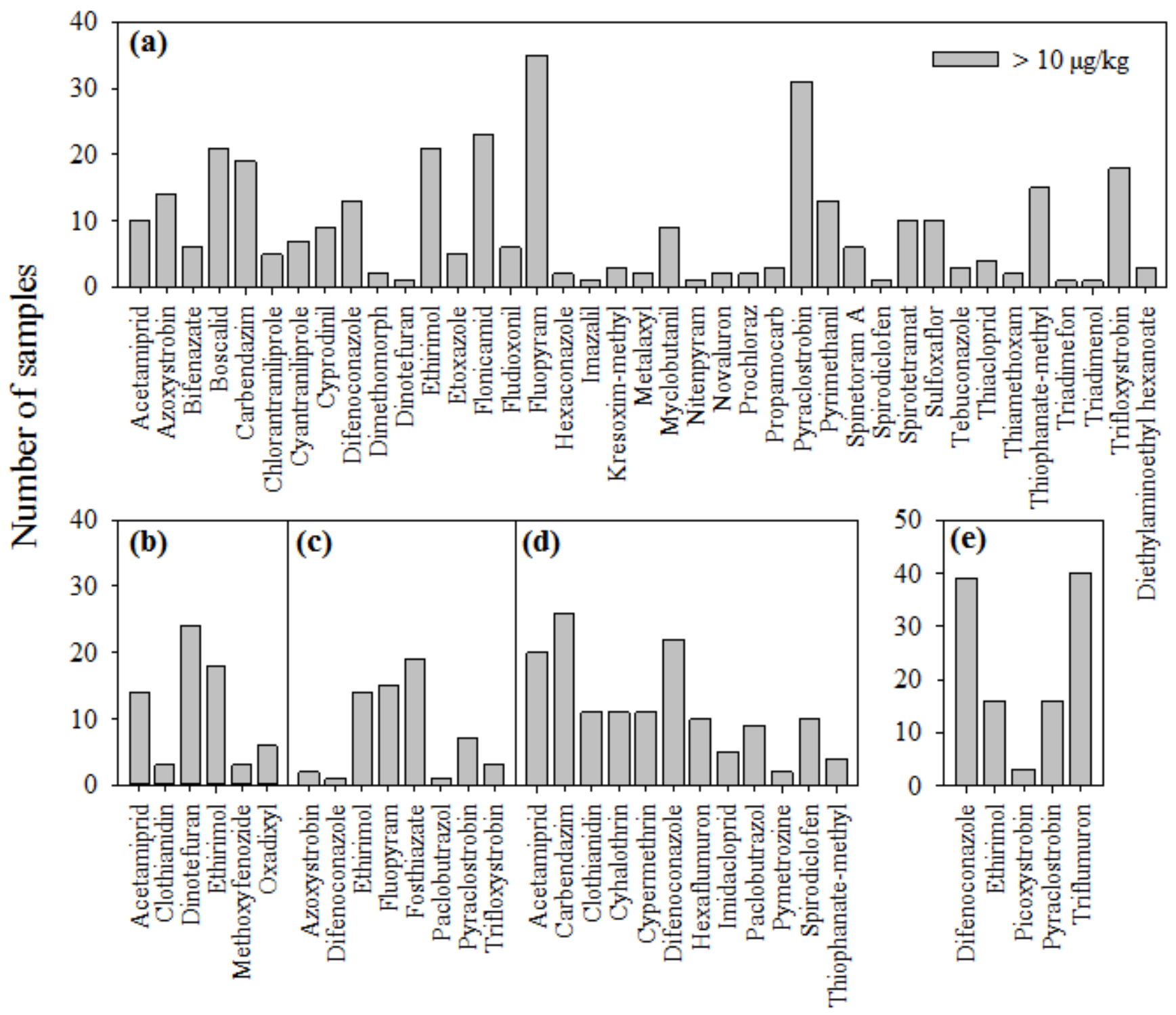

Figure 2

Number of samples with different pesticide residues in strawberries (a), watermelons (b), melons (c), peaches (d), and grapes (e).

\section{Supplementary Files}

This is a list of supplementary files associated with this preprint. Click to download.

- 1Supplementarylnformation.docx 